ULRIKE HASS-ZUMKEHR (Mannheim)

\title{
Spiegelungen der Rechtssprache in der Lexikographie ${ }^{1}$
}

Alte Sprachwörterbücher und auch Sachenzyklopädien sind heutzutage in allen Disziplinen als Kulturdokumente vergangener Epochen beliebt, weil sich, so heißt es oft, die Vorstellungen einer Zeit in ihnen spiegeln. Es scheint ein Leichtes, je nach Untersuchungsziel dort explizite Aussagen zu den in Frage stehenden Ideen aufzufinden. Erfahren wir also, wie „man“ im 18. Jahrhundert über das Recht gedacht hat, wenn wir in den Wörterbüchern dieser Zeit die sinn- und sachverwandten Stichwörter hierzu aufschlagen? Nein, so einfach ist es nicht - der ZeitSpiegel Wörterbuch oder (Sach-)Lexikon enthält ebenso viele Brechungen wie etwa ein Reisebericht oder sonst irgendeine literarische Gattung. Wie bei diesen können auch Wörterbücher und Enzyklopädien nur dann als historische Quelle oder als Hilfsmittel zur Feststellung der Normalbedeutung benutzt werden, wenn über die Art der jeweiligen Brechung Klarheit hergestellt worden ist.

Autoren und Adressaten der Wörterbücher stehen in einer Diskussion über den aktuellen Zweck und die gesellschaftliche Aufgabe ihrer Arbeit, so daß uns aus diesen Werken heute keineswegs eine glatte Abbildung des Denkens und Handelns früherer Epochen entgegenleuchtet, sondern immer eine Mischung aus Dokumentation und versuchter Einflußnahme auf die Sprache und die Weltsicht der Benutzer. $^{2}$ Enzyklopädien stehen ebenfalls, aber in partiell anderen, Diskussionen und Diskursen; ich beschränke mich hier auf sprachbezogene Lexika, d.h. auf Wörterbücher.

Die vom Veranstalter zitierte Generalinstruction (vgl. Einleitung, S. 1) des brandenburgischen Kurfürsten vom 11. Juli 1700 steht im Kontext einer zeitlich wie personell ausgedehnten Programm-Diskussion über die Möglichkeiten der "Verbesserung der deutschen Sprache" (Leibniz). Sprachplanerische Eingriffe von staatlichen Institutionen oder gesellschaftlich einflußreichen Personengruppen waren seinerzeit nichts vollkommen neues, aber neu war, daß sich das sprachplanerische Instrumentarium nun auf das Wörterbuch konzentrierte. Mit keinem anderen Medium vor Erfindung des Computers läßt sich der Wortschatz einer Sprache

1 Da der Verlag Einheitlichkeit der Rechtschreibung verlangt, stimmte die Autorin einer Rückănderung ihres Beitrags in die alte Schreibung zu.

2 Vgl. Haß-Zumkehr, Ulrike, Der Lexikograph als Hermeneut. Über die historisch-kulturelle Gebundenheit von Wörterbüchern, in: Tommola, Hannu/Varantola, Krista/Salmi-Tolonen, Tarja/Schopp, Jürgen (Hg), EURALEX' 92 Proceedings 1-II. Tampere/Finnland 1992, S. 621629; Haß-Zumkehr, Ulrike, Die kulturelle Dimension der Lexikografie am Beispiel der Wörterbücher von Adelung und Campe, in: Gardt, Andreas/Haß-Zumkehr, Ulrike/Roelcke, Thorsten (Hg.), Sprachgeschichte als Kulturgeschichte. Berlin 1999, S. 247-266. 
so gut sammeln, ordnen, vergleichen und kommentieren wie mit alphabetisch oder auch sachlich geordneten Listen.

Zu diesem kognitiven Nutzen der Wörterbücher tritt aber noch ein autoritativer: In der Sicht der Benutzer stellen Wörterbücher immer irgendeine sprachliche Norm dar, die in Zweifelsfallen entscheidet. ${ }^{3}$ Diese Funktion hatte in der humanistischen Schullexikographie und auch in den Werken der barocken Sprachgesellschaften eine feste Tradition ausgebildet. Die Textsorte Wörterbuch entspricht den gesellschaftlichen Ordnungs- und Orientierungsbedürfnissen in sprachbezogener Hinsicht wie keine andere. Festzuhalten ist, daß die ideale Verbindung von kognitiver und autoritativer Funktion das Wörterbuch bis in die Gegenwart zum bevorzugten Instrument der Sprachplanung werden ließ. Es scheint sogar, daß dies um 1700 in besonderer und zum Teil neuartiger Weise entdeckt wurde:

Also hat es der Verfertiger gegenwărtigen Wörter-Buchs gewagt, und seine Arbeit herausgegeben, ohngeachtet viele daran gezweifelt, ob es so weit zum Stand kommen werde. Es hat ihm die $\lambda \varepsilon \xi$ เко $\nu_{\imath} \alpha$, oder Liebe zum Lexicon-Schreiben so vieler gelehrten Leute, ja sogar die $\lambda \varepsilon \xi$ เко $\mu \alpha v \_\alpha$ oder damit vorgehende Raserey dieses Seculi allerdings viel geholfen, und die Arbeit etwas erleichtert. ${ }^{4}$

Der dies schrieb, Johann Leonhard Frisch, war seit 1706 Mitglied der Preußischen Societät der Wissenschaften, stand mit Leibniz in Korrespondenz und setzte die obrigkeitlichen Aufforderungen zu Wortschatzsammlung zwecks Verbesserung der „teutschen Haubtsprache“ in die Tat um.

Die allgemeine lexikographische „Raserey“ bezog sich aber nicht nur auf die allgemeinsprachliche, d.h. auf die überregionale Standardsprache zielende Lexikographie, sondern auch auf die Lexikographie der Mundarten, die ab Mitte des 18. Jahrhunderts zuerst in Niederdeutschland zu entstehen beginnt, und jener der beruflichen Sondersprachen (,Seemanns-“, „Kaufmanns-“, „Bergmannssprache“) ab $1712 .^{5}$ In allen Fällen ist als eines unter mehreren Motiven immer auch die Behebung juristischer Verständigungsprobleme anzunehmen; die lexikographische Parallelisierung mundartlicher bzw. funktional spezifischer Rechtswortschätze beförderte nicht nur den Sprach-, sondern auch den Rechtsausgleich im Deutschen Reich.

Ich möchte im folgenden an diesem und zwei weiteren deutschen Wörterbüchern des 18. und beginnenden 19. Jahrhunderts zeigen, wie sich die sprachplane-

3 Ripfel, Martha, Die normative Wirkung deskriptiver Worterbücher, in: Hausmann, Franz Josef/Reichmann, Oskar/Wiegand, Herbert E./Zgusta, Ladislav (Hg.), Wörterbücher. Ein internationales Handbuch zur Lexikographie. Berlin 1989. 1. Teilbd., S. 189-208.

4 Frisch, Johann Leonhard, Teutsch-Lateinisches Wörter-Buch. Nachdruck der Ausg. Berlin 1741 mit e. Einf. u. Bibliographie hg. von Gerhardt Powitz. Hildesheim 1977, „Vorbericht“, S. XIV v.

5 Vgl. Kühn, Peter/Püschel, Ulrich, Die deutsche Lexikographie vom 17. Jahrhundert bis zu Grimm ausschließlich, in: Hausmann/Reichmann/Wiegand/Zgusta (Hg.), Wörterbücher, (wie Anm. 3). Berlin 1990. 2. Teilbd., S. 2049-2078. 
rischen Ideen von Leibniz bis zur Pädagogik der Spătaufklärung auf die Behandlung der Rechtssprache ausgewirkt haben. Es handelt sich außer dem erwähnten Werk von Frisch um das Grammatisch-Kritische Wörterbuch der Hochdeutschen Mundart von Johann Christoph Adelung, d.h. um die 2. bearbeitete Auflage dieses Werks von 1793-1801.6 Und drittens um das in kritischer Opposition zu Adelung entstandene Wörterbuch der deutschen Sprache von Joachim Heinrich Campe, dem Pädagogen, Volksaufklärer und Fremdwortpuristen. Dieses fünfbändige Werk erschien 1807-1811. ${ }^{7} \mathrm{DaB}$ auch diese spät datierten Wörterbücher noch in die Aufklärung hineingehören, läßt sich anhand ihrer Methodik erweisen und erklärt sich auch aus der langen Vorbereitungszeit der immer umfangreicheren Werke.

Generell werden im Wortschatz der Rechtssprache diverse Bereiche oder Schichten unterschieden; ich greife hier exemplarisch auf neuere Arbeiten von Schmidt-Wiegand zurück. ${ }^{8}$ Üblicherweise geht die Klassifikation dabei von den Entstehungszeiträumen der Wörter im Zusammenhang mit wesentlichen Rechtsentwicklungen aus. Man könnte demnach die Behandlung des Germanischen Rechtswortschatzes, des Rechtswortschatzes des frühen, hohen, späten Mittelalters usw. in den Wörterbüchern untersuchen. Dagegen spricht die sehr andere Sicht des 18. Jahrhunderts auf Geschichte und Wortgeschichte. Im Unterschied zu dieser genetischen Klassifikation habe ich deshalb für meine lexikographische Untersuchung Kriterien in den Mittelpunkt gestellt, die im sprachreflexiven Horizont der Wörterbuchautoren des 18. Jahrhunderts lagen. Hiernach muß sich der Rechtswortschatz einordnen lassen in außerhalb und innerhalb der Gemeinsprache stehende und in uberlappende Bereiche, linguistisch gesprochen in fachinterne, fachexterne und allgemeinsprachliche. In der Sicht der damaligen Lexikographen spielten hierbei die Entlehnungen aus dem Lateinischen, Französischen und sogar schon aus dem Englischen eine große Rolle; fremde Wörter wurden einheimischen in differenzierter Weise gegenübergestellt.

6 Adelung, Johann Christoph, Versuch eines vollständigen grammatisch-kritischen Wörterbuchs der hochdeutschen Mundart, mit beständiger Vergleichung der übrigen Mundarten, besonders aber der oberdeutschen. 5 Bde. Leipzig 1775-1786; ders., Grammatisch-kritisches Worterbuch der hochdeutschen Mundart. Leipzig 1793-1801. Nachdruck hg. und mit einer Einl. versehen v. Helmut Henne. Hildesheim 1975; Henne, Helmut, Einfürung und Bibliographie zu Johann Christoph Adelung, Grammatisch-kritisches Wörterbuch der Hochdeutschen Mundart (1793-1801), in: ders. (Hg.), Deutsche Wörterbücher des 17. und 18. Jahrhunderts. Einführung und Bibliographie. Hildesheim 1975, S. 109-142.

7 Campe, Joachim Heinrich, Wörterbuch der deutschen Sprache. 5 Bde. Braunschweig 18071811. Nachdruck mit einer Einf. und Bibliogr. hg. v. Helmut Henne. Hildesheim 1969.

8 Schmidt-Wiegand, Ruth, Deutsche Sprachgeschichte und Rechtsgeschichte bis zum Ende des Mittelaiters (Artikel Nr. 5), in: Besch, Werner/Betten, Anne/Reichmann, Oskar/Sonderegger, Stefan $(\mathrm{Hg}$.), Sprachgeschichte. Ein Handbuch zur Geschichte der deutschen Sprache und ihrer Erforschung. 1. Teilbd., 2. Aufl. Berlin 1998, S. 72-87; dies., Deutsche Sprachgeschichte und Rechtsgeschichte seit dem Ausgang des Mittelalters (Artikel Nr. 6), in: Besch/Betten/ Reichmann/Sonderegger (Hg.), Sprachgeschichte, (wie oben), S. 87-98. 
Außer der Unterscheidung deutsch vs. fremdsprachlich lag auch das Kriterium mündlich vs. schriftlich im Horizont der Lexikographen. Die Lexikographie des Mittelalters und des Humanismus war zwar bei den deutschen Lemmata notgedrungen mündlichkeitsorientiert gewesen; man ging aber schon in der Barockzeit dazu über, sich auf schriftliche Zeugnisse zu stützen. ${ }^{9}$ Insgesamt blieb die Wortschatzdarstellung bis zu Matthias Kramers deutsch-italienischem Wörterbuch von $1700 / 1702^{10}$ an die mündliche Individualkompetenz der Lexikographen gebunden. Erst 1734 begann mit Steinbachs deutschem Wörterbuch ${ }^{11}$ die systematische Belegung des Wortgebrauchs durch nachgewiesene deutschsprachige Texte. In der gesamten Aufklärungszeit nahmen die Spuren der Mündlichkeit in den Wörterbüchern weiter $a b$, so daß bei Jacob Grimm der Hörbeleg nur noch Notnagel war, der die Lücken der Exzerption überdecken mußte.

Vor diesem Hintergrund der Unterscheidung von deutsch vs. fremd und dem Bemühen um Quellenfundierung kann man in der Lexikographie der Aufklärung vier Klassen rechtssprachlicher Ausdrücke zugrundelegen - ich nenne nachfolgend nur die Simplizia, untersucht wurden aber immer auch alle rechtserweiternden Ableitungen und Zusammensetzungen:

1. Die rechtsbezüglichen Ausdrücke in der Gemeinsprache wie Recht, Gerechtigkeit, Strafe, Gnade, Schuld; ${ }^{12}$ sie sind rein äußerlich gesehen alle deutschen Ursprungs und nicht exklusiv schriftlich.

2. Ausdrücke, die fast ausschließlich der juristisch-gelehrten Kommunikation angehören und damit überwiegend lateinischen und schriftsprachlichen Ursprungs sind: corpus iuris, ius publicum, ius naturae oder Naturrecht, Statuten, Polizei.

Ferner Ausdrücke, die sowohl dem juristischen wie dem allgemeinen oder öffentlichen Sprachgebrauch angehören. Dies ist z.B.

3. beim Prozeßrecht und

4. beim Verfassungsrecht teilweise der Fall.

Die prozeßbezüglichen Ausdrücke wie Prozeß, Richter, Gericht, Anwalt, Schöffe, Fall, Akte, Zeuge haben neben der lateinischen eine längere deutschsprachige und auch mündliche Tradition. Im Unterschied dazu haben verfassungsrechtliche Aus-

9 Stieler mußte in diesem Punkte aber noch hinter Schottels Forderungen zurückbleiben; vgl. Schmidt-Wiegand, Art. 6, (wie Anm. 8), Abschnitt 2.

10 Kramer, Matthias, Herrlich-großes Teutsch-Italiänisches Dictionarium. 2 Bde. 2. Auflage 1700 bis 1702 (1. Auflage 1678). Nachdr. mit e. Einf. hg. von Gerhard Ising. Hildesheim 1982.

11 Steinbach, Christoph Ernst, Vollständiges Deutsches Wörterbuch. Nachdruck der Ausg. Breslau 1734. Nachdr. mit einer Einf. hg. v. Walther Schröter. Hildesheim 1973.

12 Nach sprachwissenschaftlichem Usus werden in diesem Beitrag objektsprachliche Erwähnungen von Wörtern kursiv und Bedeutungsangaben in einfachen Anführungszeichen gesetzt. 
drücke wie Reich, Staat, Verfassung, Fürst, Volk, Souverän, absolut, Gewalt, Untertan im späten 18. Jahrhundert inhaltsseitig und z.T. auch ausdrucksseitig englische und französische Traditionen aufgenommen. Sie kennzeichnen aber neuere, nicht mehr nur rechtliche, sondern auch politische Entwicklungen, die den Weg ins 19. und 20. Jahrhundert weisen. ${ }^{13}$

Es geht im folgenden um die Fragen, wie die Lexikographen der Aufklärungszeit den juristischen und den allgemeinen Gebrauch dieser Wörter darstellten und womöglich in ein Verhältnis zueinander brachten, wo und warum sie sprachplanerisch eingriffen, ob solche Eingriffe puristisch auf die Wortform bezogen blieben oder sich auch auf die begrifflichen Inhalte erstreckten.

\section{Johann Leonhard Frisch}

Das Teutsch-Lateinische Wörterbuch von Frisch ist ein Wörterbuch, das die gesamte deutsche Sprache erfassen wollte. ${ }^{14}$ Hinter ihm steht der Anspruch, der großen Vielfalt der sprachlichen Verkehrsformen, in denen sich das Deutsche auch noch zu Beginn des 18. Jahrhunderts darstellte, eine alphabetische Ordnung zu geben und so die Integration der regionalen, beruflich-funktionalen, gattungsgebundenen und auch der vergangenen alten Sprachformen voranzutreiben. Frisch zăhlt sie in seiner langen Titelei alle auf (siehe Anhang). Auch das Lateinische, das hier traditionsgemăß als eine Art Tertium comparationis mit den deutschen Varietäten parallelisiert wird, ist in der ersten Hälfte des 18 . Jahrhunderts ja immer noch ein faktisches Kommunikationsmittel, vor allem in den Wissenschaften.

Frisch bezieht sich mit seinem Wörterbuchkonzept explizit auf Leibniz' Ideen, wie sie in den Unvorgreiflichen Gedanken betreffend die Ausübung und Verbesserung der deutschen Sprache von 1697 entwickelt wurden. ${ }^{15}$ Man kann sein Wörterbuch mit Recht als einen Versuch betrachten, nicht nur einen landschaftlichen Sprachausgleich zu befördern, sondern auch die Sprache der Gelehrten durch Aufnahme der alten „echt" deutschen Wörter zu verbessern, d.h. von fremdsprachlichen Interferenzen zu befreien und ausdrucksseitig "patriotischer" zu machen. Leibniz dachte dabei an mehrere getrennte Wörterbücher:

${ }^{13}$ Hilfreich bei der Auffindung der Wortschätze waren: Stein, Peter G., Römisches Recht in Europa. Die Geschichte einer Rechtskultur. Frankfurt 1996; Wesel, Uwe, Geschichte des Rechts. Von den Frihtermen bis zum Vertrag von Maastricht. München 1997.

14 Reichmann den Frühformen bis zum Vertsiks in: Hausmann/Reichmann/Wiegand/Zgusta (Hg.), Wörterbilcher, 1. Teilbd., (wie Anm. 3),

S. 230-246, insb. S. 236.
Leibniz, Gottfried Wilhelm, Unvorgreifliche Gedanken betreffend die Ausibung und Verbesserung der deutschen Sprache. Zwei Aufsätze, hg. v. Uwe Pörksen, kommentiert von Uwe Pörksen und Jürgen Schiewe. Stuttgart 1995. 
nämlich ein eigenes Buch für durchgehende Worte, ein anderes für Kunstworte und letzlich eines für alte und Landworte und solche Dinge, so zur Untersuchung des Ursprungs und Grundes dienen, deren erstes man Sprachbrauch, auf lateinisch Lexikon, das andere Sprachschatz oder cornu copiae, das dritte Glossarium Etymologicum oder Sprachquell nennen möchte. ${ }^{16}$

Frisch nun vereinigt alle in einem Band und nutzt Leibniz' Unterscheidungen zwischen allgemeinem, berufs- und landschaftsspezifischem Gebrauch und spracharchäologischen Erläuterungen lediglich zur Gliederung seiner Wortartikel. Dem Sprachausgleich wird dadurch viel besser gedient, da $\beta$, recht ${ }^{*}$ in der Bedeutung von, gut und richtig ' unmittelbar neben der Bedeutung ,rechtmäßig ‘ erläutert wird, statt in einem anderen Buch. Tatsächlich wäre die Rechtssprache nach Leibniz' Vorschlag wohl auf alle drei Bücher verteilt worden, denn sie läßt sich eben nicht als eine spezifische Berufssprache behandeln wie die von Bergbau und Fischerei, und sie läßt sich auch nicht entweder dem allgemeinen Gegenwartsgebrauch oder dem Gebrauch früherer Epochen zuordnen, sondern nur in der Verbindung von Geschichte und Gegenwart darstellen.

Wie sah nun die Behandlung des Rechtswortschatzes in Frischs Wörterbuch de facto aus? Am folgenreichsten erwies sich, daß er - auch hierin einem Vorschlag Leibniz' folgend - in großem Umfang ältere Rechtsquellen aus allen deutschen Landschaften exzerpiert hatte. Sein dreiseitiges Quellenverzeichnis enthält über 400 Titel, von denen schätzungsweise die Hälfte Rechtsquellen im engeren Sinne sind. Man findet etwa ein Bayerisches Jagd- und Fischrecht, die „Clevische Policey-Ordnung“, die „Peinliche Hals-Gerichts-Ordnung“, eine Rostockische KleiderOrdnung von 1585, eine Preußische Bernsteins-Ordnung von 1693, aber auch rechtsgelehrte Schriften, etwa von Gobler. Neben Rechtsquellen sind Chroniken, Reisebeschreibungen und unter anderem sehr viele naturkundliche Werke vertreten.

Frischs Quellenmasse stammt aus dem 15. bis 17. Jahrhundert, ${ }^{17}$ d.h. nur zu einem geringen Teil aus Frischs unmittelbarer Gegenwart. Rechtsgeschichtlich wird damit die gesamte Zeit der Rezeption abgedeckt, die für die Entwicklung des deutschen Rechtswortschatzes entscheidend war. Frischs Interesse an den Rechtsquellen richtete sich aber nicht primär auf ihre rechtliche Seite, sondern auf den in ihnen enthaltenen Wortschatz der zum Teil abgelegenen Berufe und Sachbereiche, ${ }^{18}$ der in diesen Ordungen und Statuten oft erstmals verschriftlicht worden war. Um der "voces Technica“ willen habe er „so viel Teich-Ordnungen, als er habe können, durchlesen, und die darin angemerkte wörter, so bei dieser sache ge-

16 Leibniz, (wie Anm. 15), S. 18.

17 Powitz, (wie Anm. 4), Einleitung, S. XII**.

18 Ebd. 
braucht werden, ausgezogen", so steht es in einem Protokoll der Berliner Societät von 1727. ${ }^{19}$ Eine gesonderte Gattung sah er in den Rechtsquellen also nicht.

Diese Art der Auswertung älterer deutscher Rechtstexte führt in Frischs Wörterbuch dazu, daß der Gebrauch eines Worts nur selten mittels der Formulierung „in den Rechten“ oder „im juristischen Stylo"20 markiert wird. Dem entspricht, daß im Register der lateinischen Wörter kaum rechtsspezifische Ausdrücke vorkommen: corpus ja, corpus juris nein, die lateinischen Bezeichnungen für Floßrecht und Fährgerechtigkeit ja, die für Naturrecht nein.

An den rechtsbezüglichen Ausdrücken in der Gemeinsprache hebt Frisch auffallend oft und lobend ihr Alter hervor und zeigt darin ein Fortwirken der barocken Wörterbuchtradition. Der Wortartikel Schultz z.B. stellt eine kleine Rechtsgeschichte dieses Amtes dar, in der rechtliche Sachverhalte mehrmals mittels ehemals, anfänglich und in den alten Gesetzen kommentiert und somit deutlich von den Amtsbräuchen des Schulzen in der Gegenwart unterschieden werden, wenn es etwa heißt: „ist noch in Sonderheit auf den Dörfern gewöhnlich““ ${ }^{21}$

Der positive Bezug auf die Vergangenheit bei Leibniz wie bei Frisch ähnelt zwar demjenigen der barocken Sprachpflege, unterscheidet sich aber von jener durch den rationalen Zugriff auf die Zeugnisse. Die spekulativen Etymologien seiner Vorgänger geht Frisch im Vorbericht seines Wörterbuchs hart an:

Wo die Etymologie gar ausgelassen ist, hat sie der Verfasser nicht gewußt. Man will hier lieber eine behutsame Unwissenheit bekennen, als ein verwegenes Wissen vorgeben. [...] Kurtz zu sagen, [...] man hat in Ansehung aller erstgemeldten Ursachen, denjenigen für einen Ertz-Praler zu halten, der da sagt, er wisse wo alle unsere Wörter herkommen. ${ }^{22}$

Es scheint, daß das besondere Alter vieler Rechtswörter ausschlaggebend für ihre ausfuhrliche Behandlung durch Frisch war, selbst wenn Wortform oder -inhalt in der ersten Hälfte des 18. Jahrhunderts nicht mehr verwendet werden. Gleiches trifft auch auf den biblischen Wortschatz Luthers zu; auch hier verzichtet Frisch nicht auf inzwischen Veraltetes, denn er verfolgt mit seinem Wörterbuch auch die Absicht, den Zugang zu diesen alten Texten offen zu halten. ${ }^{23}$

Aus der Schwerpunktsetzung bei den Rechtsquellen und der besonderen Hervorhebung des Alters der Wörter, gleich aus welchen Quellen sie stammen, resultiert ein Wörterbuch, in dem diverse Einzelwortschätze erstmals miteinander verbunden, verglichen und disparate Verwendungsweisen eines Worts eng aufeinander bezogen werden. Dies gelingt durch einen methodischen Fortschritt, denn

19 Zit. ebd.

${ }^{20}$ Frisch, (wie Anm. 4), S. 5.

21 Frisch, (wie Anm. 4), S. 233; ăhnlich ebd. im Wortartikel Schöpfe.

${ }_{22}$ Frisch, (wie Anm. 4), Vorbericht S. X1 r/v.

23 Die Lexikographie sollte die Brücke schlagen helfen zu den immer schwerer verstăndlichen alt-, mittel- und sogar frühneuhochdeutschen Texten; in den Schriften kurz vor und kurz nach der Erfindung des Buchdrucks stünden, so Frisch, ,auf allen Seiten Wörter [...] die dem Leser am Verstand solcher Schrifften hinderlich fallen", (wie Anm. 4), Vorbericht, S. X2 r. 
Frisch hebt die ausführliche semantische Kommentierung auf ein lexikographiegeschichtlich neues Niveau. Allerdings gewinnt er den gemeinsprachlichen Teil seiner Lemmata nicht aus Textquellen, sondern aus den Wörterbüchern seiner jüngsten Vorgänger Steinbach (1734) und vor allem Matthias Kramer (1700/ $1702)^{24}$ und tradiert somit auch eine Schicht mündlicher Rechtssprache in die Lexikographie des 18. Jahrhunderts. Von Kramers Beispielen gesprochener Sprache übernimmt Frisch z.B. einen Proce $\beta$ haben, gewinnen, verlieren ${ }^{25}$ und variiert dies noch mit einem einen Proce $\beta$ an den Hals werfen; allerdings wird Rechtshandel durch Proce $\beta$ ersetzt (Frisch s.v. Proceß).

Die Integration funktional unterschiedlicher Wortschätze bei Frisch gilt für die rechtlich relevanten Wörter der Gemeinsprache und für die allgemein bekannten alten deutschen Rechtswörter. Ausgeschlossen aus diesem Fokus bleiben der lateinisch gebildete gelehrte Rechtswortschatz und der Mischbereich des Verfassungsrechts als eines seinerzeit noch schmalen und öffentlich irrelevanten Rechtswortschatzes. Beide werden nicht nur kaum in die Gesamtsprache integriert, sondern sind bei Frisch überhaupt auffallend schwach repräsentiert. Zu Völkerrecht etwa wird nur das Äquivalent jus gentium verzeichnet, und in den Artikeln Fürst oder Volk fehlt jeder Rechtsbezug; stattdessen wird über die angemessenen Anredeformen und Titulaturen informiert.

Ein Beispiel für die integrative Behandlung rechtsspezifischer und gemeinsprachlicher Bedeutungen ist der Artikel Kämerer. Genannt werden hintereinander erstens der „Camerarius, auf den Rathhäusern, quaestor" usw., zweitens „bei Königen und Fürsten für Kammerherr, cubicularius, nobilis primi ordinis", drittens „In der Bibl. Ubersetzung, eunuchus, spado, cubicularius“ und schließlich viertens "Camerierer, in der Pommerisch. Holz- und Jagdordn. p.[pagina] 29. Noch vor dem Rent-Meister, nach dem Amts-Hauptmann". ${ }^{26}$ Die Integration bezieht sich, wie man sieht, selbstverständlich auch auf die landschaftlichen Unterschiede im Wortgebrauch; zwar homogenisiert Frisch diese noch nicht, liefert aber durch seine ordnende und parallelisierende Arbeit die Voraussetzungen für etwaige sprachpolitische Eingriffe in Richtung auf den regionalen Sprachausgleich. Solche Variantenlisten konnten auch die Wortwahl bei der Formulierung überregionaler Gesetze anleiten.

Diejenigen Wörter, bei denen sich rechts- und gemeinsprachliche Bedeutung vermischen, werden von Frisch vielleicht noch integrativer dargestellt, als es der damaligen Sprachwirklichkeit entsprach. Eine Ausnahme stellen die thematisch zentralen Wörter Recht (Subst.), recht (Adj.) und richten dar, für die Homonyme angesetzt werden entsprechend equum, bonus gegenüber jus, dirigere gegenüber

24 Powitz, (wie Anm. 4), Einleitung, S. XI*; Powitz, Gerhardt, Das deutsche Wörterbuch Johann Leonhard Frischs. Berlin 1959, S. 22 f.

25 Kramer, (wie Anm. 10), Bd. II, S. 280, 281

26 Frisch, (wie Anm. 4), S. 498. 
judicare. Die integrative Darstellung von Gemeinsprache und Rechtssprache überwiegt jedoch, so daß man festhalten kann: Frisch hat die deutsche Rechtssprache des 15 . bis 17. Jahrhunderts umfassend im allgemeinsprachlichen Wortschatz etabliert und durch seine integrative Darstellung ein lexikographisches Instrument geschaffen, das gut geeignet war, Leibniz' Vorstellungen von der Verbesserung auch der Juristensprache zu unterstützen.

Übrigens käme eine Untersuchung der theologischen und kirchlich-institutionellen Sprache bei Frisch zu einem sehr ähnlichen Ergebnis; die älteren deutschen Bibelübersetzungen haben den gleichen Quellenstatus wie die alten Rechtsquellen, während die wissenschaftlich-theologische Literatur in etwa derjenigen der juristisch-gelehrten entspricht. Die juristische Ausrichtung des Wörterbuchs ist allerdings deutlich stärker als die theologische.

Johann Leonhard Frisch stammte aus einer protestantischen Theologen- und Juristenfamilie der bayerischen Oberpfalz. Nach dem Theologiestudium trieb er sich einige Jahre herum als Erzieher im Harz, im Odenwald, als Dolmetscher auf dem Balkan und als landwirtschaftlicher Hofverwalter nahe Nürnberg. Schon in dieser Zeit hat er sich für Natur- und Sprachdinge interessiert und mit Leibniz korrespondiert. Mit 32 Jahren kam er als Gymnasiallehrer, später Rektor in Berlin äußerlich zur Ruhe. Hier arbeitete er an Lehrbüchern und Übersetzungen und publizierte Wissenschaftliches über die Seidenraupenzucht, über Insekten überhaupt, über Vögel, über Farbenchemie, über die ältesten deutschen Wörterbücher, und vieles mehr. Als der preußische König Friedrich I., vormals Kurfürst von Brandenburg, ihn mit der Seidenproduktion beauftragte, ließ Frisch auf allen Berliner Grünflächen Maulbeerbäume anpflanzen. Erfolgreich war auch sein verbessertes Herstellungsverfahren des Farbstoffs „Berliner Blau“. Neben allen diesen Aktivitäten arbeitete er rund 50 Jahre lang an seinem Wörterbuch. ${ }^{27}$

Powitz hat Frischs exakt beschreibende Darstellungen naturkundlicher Gegenstände mit seiner lexikographischen Methodik zur Deckung zu bringen versucht Sammeln und Ordnen hier wie dort. ${ }^{28}$ Empirie und Deskriptivität sind in der Tat die herausragenden und lexikographiegeschichtlich neuen Merkmale von Frischs Wörterbuch.

${ }_{28}^{27}$ Powitz, (wie Anm. 24), S. 1.

28 Zur gleichen Zeit, da er die Sammlungen seines Insekten- und Vogelkabinetts Stück um Stück zu vervollständigen sucht, trägt er unermüdlich exzerpierend wortgeschichtlichen Erfahrungsstoff aus den Quellen zusammen, um Einblick zu gewinnen in die Sprache als Erscheinung der objektiven Wirklichkeit." Powitz, (wie Anm. 24), S. 9. 


\section{Johann Christoph Adelung}

Machen wir nun einen Sprung von gut 50 Jahren. In der zweiten Hälfte des 18. Jahrhunderts entstand eine neue Programmdiskussion um Art und Zweck des Wörterbuchs, diesmal getragen von den Schriftstellern. Im Zentrum ihrer Vorstellungen stand nicht mehr eine Bestandsaufnahme des Deutschen in seiner Vielfalt, sondern wiederum seine Verbesserung, nun allerdings für Zwecke der Dichtung: Stilsicherheit, Ausdrucksreichtum und Flexibilität waren die Ziele, denen das Wörterbuch jetzt dienen sollte. Lessing, Klopstock und Wieland forderten eines, das die Sprache der schönen Literatur dokumentiert und ihr so einen Einfluß auf die Gemeinsprache sichern würde. Gottsched begann mit den Arbeiten für solch ein Wörterbuch, aber abgeschlossen wurde es in einer Form, die nicht unerheblich von den programmatischen Ideen der Dichter abwich: Johann Christoph Adelungs Versuch eines grammatisch-kritischen Wörterbuchs der Hochdeutschen Mundart mit beständiger Vergleichung der übrigen Mundarten, besonders aber der oberdeutschen erschien in fünf Bänden von 1774 bis 1786 in Leipzig. Der Titel verrät, daß nun eine der regionalen Varietäten als besonders geeignet und vorbildlich kodifiziert werden sollte. Tatsächlich handelte es sich um eine regional wie sozial bestimmte, d.h. auf höfische Kreise beschränkte Varietät. Adelungs Bevorzugung der mündlichen Umgangssprache der ,obern Classen“ Obersachsens oder Meißens erregte heftige Kritik, als deren Folge von 1793 bis 1801 eine zweite überarbeitete Fassung mit dem Titel Grammatisch-kritisches Wörterbuch der Hochdeutschen Mundart in vier Bänden erschien.

Es blieb dennoch das Wörterbuch einer Hochsprache, die den Benutzern als allgemeiner Standard anempfohlen wurde. Adelungs Intention bestand darin, eine sprachliche Norm darzustellen, die er von dem seiner Ansicht nach besten Sprachgebrauch einer herausgehobenen sozialen Gruppe ableitete. Kompliziert wird die Diagnose dadurch, da $\beta$ diese normative Intention die lange Vorrede beherrscht, sich aber in der Wörterbuchpraxis die Beschreibung nicht nur des vorbildlichen Gebrauchs durchsetzt. Welchen Stellenwert besaß dabei die Rechtssprache? In der Vorrede heißt es:

Besonders habe ich mir angelegen seyn lassen, die Kunstwörter aus allen Künsten, Lebensarten und Wissenschaften zu sammeln, weil viele derselben selbst eingebohrnen Deutschen unverständlich und fremd sind. Unter die Kunstwörter rechne ich auch die Namen aller besondern Gebräuche, Rechte, obrigkeitlichen Aemter u.s.f., wenn sie gleich nur in dieser oder jener Provinz allein üblich sind, weil sie doch in hochdeutschen Büchern mehrmals vorkommen, und von keinem Hochdeutschen vermieden werden können, wenn er von diesen oder jenen Dingen reden oder schreiben muß. ${ }^{29}$

Den Ausschlag für die Aufnahme fachsprachlicher Wörter gibt also ihre Schwerverständlichkeit, die hier auf ihre Regionalspezifik zurückgeführt wird. In der

29 Adelung, Versuch, (wie Anm. 6), Vorrede zur 1. Aufl., S. XIII. 
Wörterbuchpraxis werden aber auch Entlehnungen aus anderen Sprachen und damit etliche lateinische Rechtswörter erklärt. Und dies geschieht nicht nur so, daß lateinische Lemmata deutsch erläutert werden, z.B. „Das Statut [...] aus dem Latein [...] ein Gesetz, welches einer Stadt, oder einer bürgerlichen Gesellschaft gegeben, oder von derselben selbst gemacht worden [...] ehedem die Willkür, $[\ldots . .]^{“, 30}$ sondern auch so, daß bei der Erläuterung deutscher Rechtswörter das lateinische Äquivalent beigefügt wird, wie

Rechtsgelehrt [...] Wissenschaft und Fertigkeit besitzend, das Verhăltniß der menschlichen Handlungen gegen die Gesetze zu bestimmen; am häufigsten als ein Hauptwort. Ein Rechtsgelehrter [...] Im gemeinen Leben ist dafür das aus dem mittlern Lat. entlehnte Jurist sehr gewöhnlich. ${ }^{31}$

Die lateinischen Äquivalente werden aber immer dann als abzulehnend markiert, wenn mehrere deutsche Ausdrücke zur Verfügung stehen. Der Wortartikel Prozeß enthält die Anmerkung: „Dieses fremde Wort ist zugleich mit dem Römischen Rechte in Deutschland eingefuihrt worden. Vorher hatte man eigene deutsche Ausdrücke dafür". 32

Die im Vergleich zu Frisch größere Freiheit Adelungs in der Aufnahme fremdsprachlicher Entlehnungen ist einmal darauf zurückzuführen, daß die starke Vergangenheitsorientierung, die Demonstration des Uraltertums der deutschen „Haubtsprache", obsolet geworden war. Zum zweiten war in der Sprachentwicklung der zweiten Hälfte des 18. Jahrhunderts die Dominanz des Lateins endgültig überwunden worden; das Ziel einer überregionalen Varietät des Deutschen war deutlich näher gerückt, obwohl auch Adelung noch am Sprachausgleich arbeiten mußte.

Er schreibt ein dominant gegenwartsbezogenes Wörterbuch und verschiebt alle form- und bedeutungsgeschichtlichen Informationen und damit einen großen Teil der von Frisch übernommenen Angaben ans Artikelende in eine Anmerkung. Der Schwerpunkt seiner Quellenbasis liegt bei literarischen Texten von etwa 1740 bis 1760 , sofern sie das von ihm geforderte „Hochdeutsch“ repräsentieren, aber auch die lexikographischen Vorgänger seit frühneuhochdeutscher Zeit sind extensiv eingearbeitet.

All diese Voraussetzungen führen dazu, daß die rechtsspezifischen Wörter bzw. Wortbedeutungen in Adelungs umfangreichem Werk eine relativ sehr viel kleinere Rolle spielen als bei Frisch. Markierungen wie ,in den Rechten“, ,in den Gerichten", „im gerichtlichen Verstande“ sind selten, und Erläuterungen wie „Straffall [...] in den Gerichten, Fälle, wo Strafen statt finden, welche bestrafet zu werden verdienen", 33 demonstrieren nicht gerade juristischen Sachverstand. Der ältere

${ }^{30}$ Adelung, Grammatisch-kritisches Wörterbuch, (wie Anm. 6), Bd. 4, Sp. 307.

31 Ebd., Bd. 3, Sp. 1008.

${ }_{32}^{32}$ Ebd., Bd. 3, Sp. 851 .

${ }^{33}$ Ebd., Bd. 4, Sp. 417 
deutsche Rechtswortschatz scheint, wie die Artikel Schuldheiß, Stab, Vogt zeigen, größtenteils in enger Anlehnung an Frisch dargestellt zu sein, einschließlich der wiederholten Markierung als veraltet. Ausschlaggebend für die Repräsentation der Rechtssprache in Adelungs Wörterbuch ist jedoch eine weit über Frisch hinausgehende Integration rechtlicher Wortverwendungen in die Gemeinsprache, die wiederum mit einer methodischen Innovation zusammenhängt.

Adelungs Verfahren der Bedeutungserläuterung ist erstens ausführlicher und differenzierter als bei seinen Vorgängern; zweitens geht er von einer relativ konstruierten abstrakten Grundbedeutung aus, von der dann die konkreten, u.a. die rechtlichen Bedeutungen abgeleitet werden. Adelung selbst sprach von einer „Bedeutungsleiter". In diesen semantisch-hierarchischen Bäumen nun sitzt das Recht regelmäßig auf den kleineren Zweigen, nicht auf den dicken Ästen. Im Wortartikel zum Substantiv Recht z.B. wird die Grundbedeutung ,Zustand, da etwas recht ist, und dasjenige, was recht ist" in zahlreiche Untergliederungen geteilt. Das positive Recht rangiert dabei unter 2.1.b) $\alpha$ :

2. „als ein Concretum“, 1) „Ein Gesetz, die Richtschnur menschlicher Handlungen“, (b) „In weiterer Bedeutung“, $(\alpha)$ „Objektive, die Sammlung der Inbegriff der Gesetze einer Art".

Diese Angabe wird dann allerdings mit der folgenden Beispielreihe illustriert: „Das göttliche Recht [...] Das geistliche, päpstliche oder kanonische Recht. Das bürgerliche Recht. [...] Das gemeine oder Deutsche Recht im Gegensatze des Römischen Rechtes." ${ }^{34}$ Juristisches taucht auch noch in weiteren Gliederungspunkten des Artikels Recht auf, statt an einer Stelle gebündelt zu werden. Im übrigen ist die Entgegensetzung von gemeinem und römischem Recht zumindest aus heutiger Sicht nicht korrekt.

Kurzum, Adelungs feine hierarchische und per Abstraktion konstruierte Bedeutungsgliederung stellt einerseits eine enge Verflechtung, ja oft eine Gleichsetzung von moralischem, biblischem und positivem Recht her, macht andererseits aber auch deren Unterscheidungen sichtbar.

Ich komme noch kurz auf den verfassungsrechtlichen Wortschatz zu sprechen. In den Wortartikeln Fürst, Gewalt, König, Reich und Staat spielen die rechtlichen Aspekte eine sehr untergeordnete Rolle. Lediglich etliche Kompositen zu Reich und Fürst, die Organe des Deutschen Reichs bezeichnen, sind meist markiert mit ,in dem Deutschen Staatsrecht“. Aber obrigkeitliche Gewalt wird unter der Bedeutung ,Herrschaft' per Beispiel in eine Reihe mit „eine Sprache in seiner Gewalt haben“ gesetzt. Die von Wolff geprägte „elterliche Gewalt" ${ }^{\text {“35 }}$ fehlt. $^{36}$

Als lexikographisches Prinzip kann festgehalten werden, daß Adelung die rechtliche Bedeutung eines Ausdrucks stets als besonderen und relativ nebensäch-

34 Ebd., Bd. 3, Sp. 1002.

35 Schmidt-Wiegand, (wie Anm. 8), Art. 6.

36 Adelung, Grammatisch-kritisches Wörterbuch, (wie Anm. 6), Bd. 2, Sp. 651 f. 
lichen Fall einordnet, der zudem erst auf späteren, jüngeren Stufen der Bedeutungsleiter entstanden sei. Trotz der rationalistischen Semasiologie sind bei Adelung keinerlei Spuren der Definitionsarbeit eines Thomasius oder Wolff zu erkennen. Die Integration der Rechts- in die Hochsprache führt Adelung weit über Frisch hinausgehend fort, so weit, daß die rechtliche Dimension des deutschen Wortschatzes relativ zur literatur- und schreibsprachlichen Dimension des Wörterbuchs nahezu verschwindet.

\section{Joachim Heinrich Campe}

Die Rechtssprache wurde aber in besonderer Weise wieder zum Vorschein gebracht durch Joachim Heinrich Campe, der sein fünfbändiges Wörterbuch der deutschen Sprache 1807-1811 in engem, aber äußerst kritischem Bezug auf den Vorgånger Adelung verfaßte bzw. verfassen ließ. ${ }^{37}$ Der vielseitige Pädagoge und Schriftsteller war Anhänger der Französischen Revolution und einer der herausragenden Verbreiter revolutionären Gedankenguts in Deutschland, und er blieb dies in der lexikographischen Arbeit. ${ }^{38}$ Er ersetzte die höfische und oberdeutsche Ausrichtung Adelungs durch bürgerliche, konstitutionell-demokratische und nationale Orientierungen. Aus diesen Vorzeichen resultieren für die lexikographische Tradierung des Rechtswortschatzes vier wesentliche Neuerungen:

Erstens streicht Campe alle Rechts- und Wortgebräuche, die schon bei Adelung als veraltet markiert worden sind, etwa bei Schuld und Schuldheiß. Dafur wird die Stichwortmenge u.a. durch die Aufnahme bürgerlichrechtlicher und verfassungsrechtlicher Ausdrücke wie Reichsverfassung, Staatsbeamter, Staatsumwälzung, Volksherrschaft, Volksversammlung erweitert. Campe versäumt dabei nicht, auf die vielfaltigen rechtlichen Folgen der Aufhebung des Deutschen Reiches hinzuweisen. Kommentare wie ,in der ehemaligen deutschen Reichsverfassung “, „ehemals im Deutschen Staatsrechte" findet man besonders oft in der Reichs-Strecke.

Er arbeitet mittels seines Wörterbuchs an der neuen Zeit, die angebrochen sei. Seine Erläuterungen verfassungsrechtlicher Ausdrücke sind daher nicht selten appellativ-utopisch. Z.B. ist der Staatsbeamte:

${ }^{37}$ Henne, Helmut, Braunschweigische Wörterbuchwerkstatt - Joachim Heinrich Campe und sein(e) Mitarbeiter, in: Schmitt, Hanno in Verbindung mit Peter Albrecht u.a. (Hg.), Visionäre Lebensklugheit. Joachim Heinrich Campe in seiner Zeit (1754-1818). Wiesbaden 1996, S. 215-224.

38 Henne, Helmut, Einfuhrung und Bibliographie zu Joachim Heinrich Campe, Worterbuch der Deutschen Sprache (1807-1811), in: ders. (Hg.), Deutsche Wörterbilicher des 17. und 18. Jahrhunderts. Einfürung und Bibliographie. Hildesheim 1975, S. 143-168; Schiewe, Jürgen, Joachim Heinrich Campes Verdeutschungsprogramm. Überlegungen zu einer Neuinterpretation des Purismus um 1800, in: Deutsche Sprache, 16. Jg. 1988, Heft 1, S. 17-33. 
[...] der Staatsdiener, sofern er dadurch dem Ganzen, wovon er ein sehr untergeordneter Theil ist, dienet oder in dessen Diensten ist. Der Vernunft gemäß ist also der Fürst, Kaiser, König \&c. der erste Staatsbeamte oder Staatsdiener, welcher nur durch den Staat und für den Staat da ist; aber nicht umgekehrt. ${ }^{39}$

Zweitens wird die verfassungsrechtliche Relevanz vieler von Adelung übernommener Stichwörter hervorgekehrt oder überhaupt erstmals verzeichnet. Kam obrigkeitliche Gewalt bei Adelung nur in einem Beispiel vor, so setzt Campe hierfür eine eigene Hauptbedeutung neu an:

4) Die Person oder die Personen, die eine gewisse Gewalt haben, mit der Mehrzahl (Pouvoir). So werden die Obrigkeiten zuweilen bürgerliche Gewalten genannt. Die gesetzgebende Gewalt der gesetzgebende Körper, die Personen, welche die Gesetze geben.

Danach erst kommen die ,himmlischen Gewalten“ in der dichterischen Sprache.40 Drittens werden alle entlehnten Wörter radikal eingedeutscht.

Und er nahm sich bestimmte Bereiche, „Fächer" vor, die ,ganz Deutsch eingekleidet $\mathrm{zu}$ werden verdienen, weil sie für alle Deutsche gehören “. Dazu rechnete Campe die „Sittenlehre“, die „Gotteslehre“ (Religion), die „Rechtssprache“, „Theile der Vernunftwissenschaft“, also der Philosophie, der „Naturlehre“ und „Naturbeschreibung“, „Theile der Arzneiwissenschaft“, die „Umgangs- und Geschäftssprache“ und die „Dichtkunst" (S. CXV ff.). Nicht ausdrücklich erwähnt ist politischer Wortschatz, den er der Rechtssprache und Philosophie zurechnen mag. ${ }^{41}$

Bevor das Wörterbuch der deutschen Sprache entstand, hatte Campe ein Verdeutschungswörterbuch verfaßt, in dem auch für viele lateinische Rechtswörter deutsche Entsprechungen vorgeschlagen wurden, die teils von ihm selbst gebildet, teils von Zeitgenossen eingefuhrt worden waren. Eine stark erweiterte Neuauflage des Verdeutschungswörterbuchs folgte 1813.42 Campes Verdeutschungsarbeit war im Unterschied zum barocken und noch bei Leibniz anzutreffenden Purismus verständlichkeitsmotiviert; Ziel war, daß auch die Bürger ohne Lateinbildung in öffentlichen Angelegenheiten mitreden können sollten. In Campes Verdeutschungswörterbuch ist der gelehrte Rechtswortschatz sehr viel breiter vertreten als je zuvor in der deutschen Lexikographie. Im Wortartikel Corpus juris heißt es zunächst: „ein Buch, worin alle (Römische) Rechte zusammengetragen sind. Man könnte vielleicht das Römische Rechtsgebäude dafür sagen“".

39 Campe, (wie Anm. 7), Bd. 4, S. 566.

40 Ebd., Bd. 2, S. 357.

41 Henne, (wie Anm. 37), S. 221, zitiert aus Campes Preisschrift von 1794.

42 Campe, Joachim Heinrich, Wörterbuch zur Erklärung und Verdeutschung der unserer Sprache aufgedrungenen fremden Ausdrücke. Ein Ergänzungsband zu Adelung's und Campe's Wörterbüchern. Neue [...] Auflage. Braunschweig 1813. 
Dann folgt ein längeres Definitionszitat von einem gewissen von Strombeck, ${ }^{43}$ in dem Pandecten, Codex und Institutionen miterläutert werden. Abschließend zitiert Campe aus einem „Heidelbergischen Verzeichniß der Vorlesungen“: „Heise erklärt die schweren Stellen des Rechtskörpers".44

Viertens dient gerade die verständlichmachende Darstellung des Rechtswortschatzes ${ }^{45}$ Campe zum Entwurf einer demokratischen Rechts- und Gesetzesidee und einer utopischen, nachabsolutistischen Gesellschaftsform. Am Schluß der umfangreichen Strecke der Ius-Kompositen schreibt er: „Man sieht, daß es uns an Rechten aller Art keineswegs mangelt; möchte uns auch eben so viel Gerechtigkeit zu Theil geworden sein oder werden! ${ }^{446}$ Erstmals wird hier in einem deutschen Wőrterbuch Kant zitiert, etwa bei gerecht, Rechtslehre und Staatenrecht. Recht, Staat und Polizei sollen nach Campes Darstellung nun für die Bürger da sein. Im Artikel Rechtlos ist von den „Rechtswohlthaten“ der „bürgerlichen Gesetze“ die Rede. Der hier erstmals lexikographisch gebuchte Rechtsanspruch wird in den Beispielen "geltend gemacht" und "durchgesetzt". Polizei ist in der ersten Bedeutung:

Die Handhabung guter Ordnung und Verfassung in einem Staate, wie auch in einer Stadt, welche besonders auf Erhaltung der offentlichen Ruhe und Sicherheit und [...] außerdem noch auf mancherlei andre die Wohlfahrt, Gesundheit, Bequemlichkeit \&c. der Einwohner betreffende Dinge Rucksicht nimmt. [...] Bei der guten Polizei hört man hier nichts von Diebereien, siehet man keine Bettler, findet man reine Straßen, die Abends hell erleuchtet sind. ${ }^{47}$

Bei Campe geht der Rechtswortschatz über in den frühen politisch-öffentlichen Wortschatz. Obwohl er auch Frisch und noch ältere Lexikographen (Stieler, Kramer) selbständig benutzt hat, scheint der ältere deutsche Rechtswortschatz weit an den Rand gedrängt. Stattdessen wird die bis dahin exklusiv gelehrte Rechtsterminologie für das Bürgertum transparent gemacht und mit Aufforderungen zur Handhabung des Rechts versehen. Einige von Campes Verdeutschungen haben sich bis heute erhalten: u.a. Bittsteller (für supplicant), Gesetzentwurf (für engl. bill), Gesetzeskraft verleihen (für sanctionieren), Gnadenfrist (indult) und - der Rechtsverdreher (chicaneur). ${ }^{48}$

${ }^{43}$ Friedrich Karl von Strombeck, 1771-1848, war in vielen Funktionen Jurist in Braunschweig, Wolfenbüttel, Kassel. Für den biographischen Hinweis danke ich Herm Christoph Leist, Heidelberg.

44 Campe, (wie Anm. 42), S. 239.

45 Kirkness, Alan, Sprachreinheit und Sprachreinigung in der Spătaufklărung. Die Fremdwortfrage von Adelung bis Campe, vor allem in der Bildungs- und Wissenschaftssprache, in: Kimfrage von Adelung bis Campe, vor allem in der Bildungs- und Wieter $(\mathrm{Hg})$, Mehrsprachigkeit in der deutschen Aufklärung. Hamburg 1985, S. 85-104, hier S. 98.

46 Campe, (wie Anm. 42), S. 389.

47 Campe, (wie Anm, 7), Bd. 3, S. 671. Die zweite Bedeutung sind die „Herren von der Polizei“; die dritte die "Polizeiwissenschaft".

48 Kirkness, Alan, Zur Sprachreinigung im Deutschen 1789-1871. Eine historische Dokumentation. Tübingen 1975, Teil 1, S. 161-167. 
Es bleibt zum Schluß die Frage nach den Folgen, die die Wörterbücher der Aufklärung für die Sprachentwicklung insbesondere der Rechtssprache und ihrer Integration in die Gemeinsprache hatten. Frischs historische Bestandsaufnahme ist bis heute wissenschaftlich unersetzlich geblieben. Von den beiden spätaufgeklärten Wörterbüchern wurde und wird Adelung als „der“ aufgeklärte Lexikograph etikettiert, obwohl er es nicht in allen Hinsichten war, während Campe bei Jacob Grimm in Ungnade fiel und seine Wörterbücher in der Folge fast wirkungslos blieben. Damit fehlt in der zentralen lexikographischen Tradition des 19. und 20. Jahrhunderts ein gemeinsprachlich sehr wichtiger Teil des Rechtswortschatzes, nämlich derjenige, der einer politisch aufgeklärten Gesellschaft die für Rechtskultur und Verfassung notwendigen Begriffe präsent gehalten hätte. 


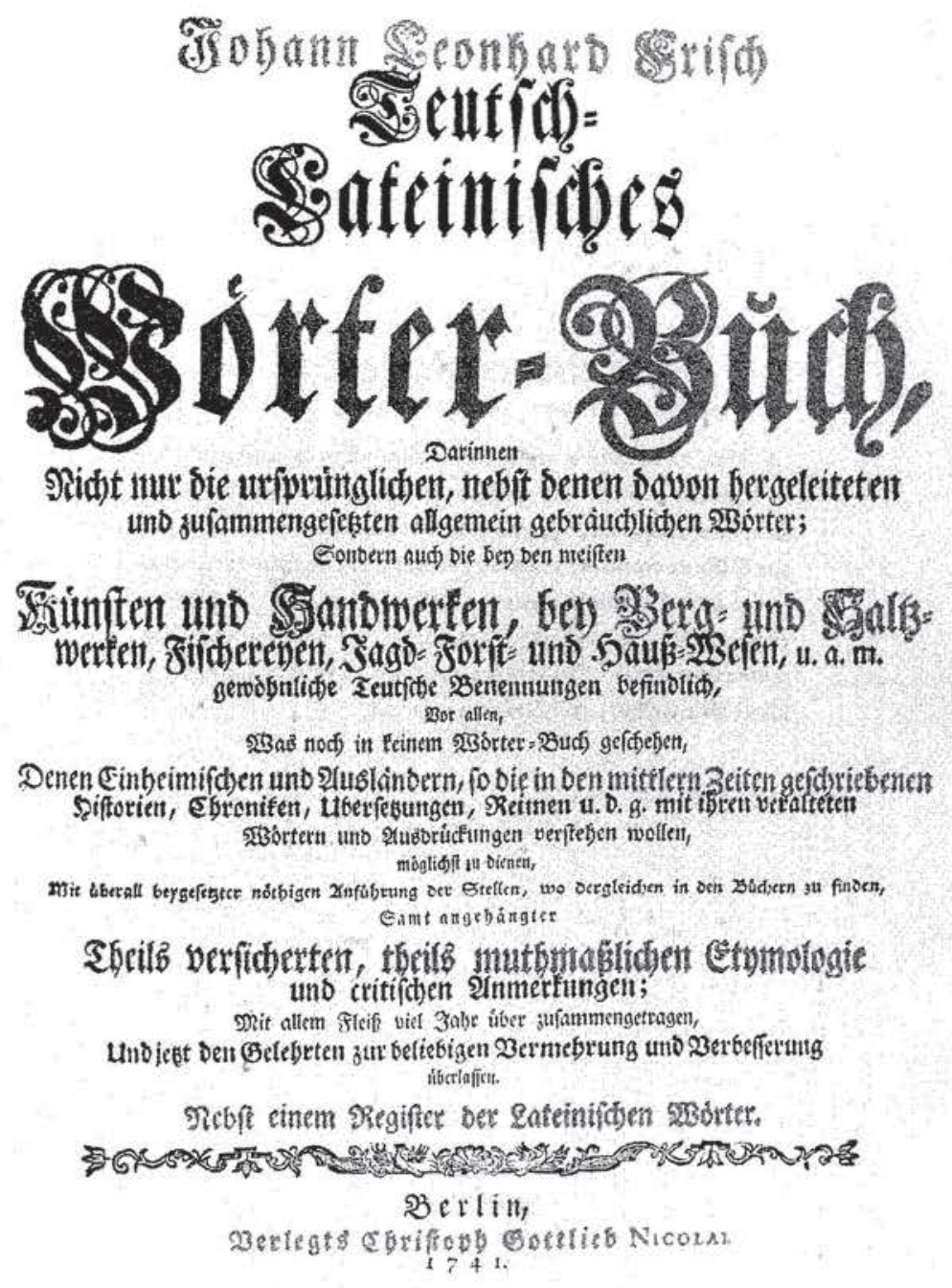

Abb. 1: Titelblatt: Johann Leonhard Frisch, Deutsch $=$ Lateinisches Wörter $=$ Buch 


\section{(G) tant atifd: ertitide}

ख

Der

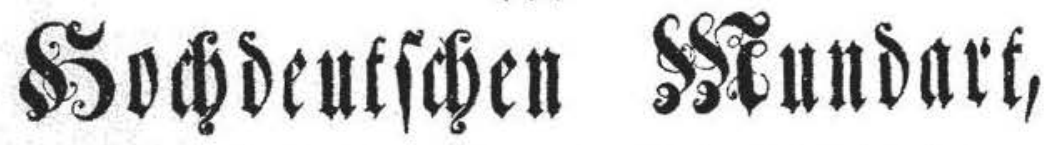

mit beftänbiger ஒergleidung Der übrfigen Nundarten, befortbers aber ber oberdentiben,

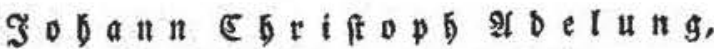

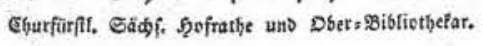

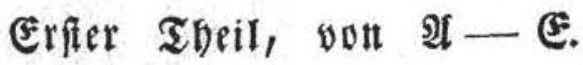

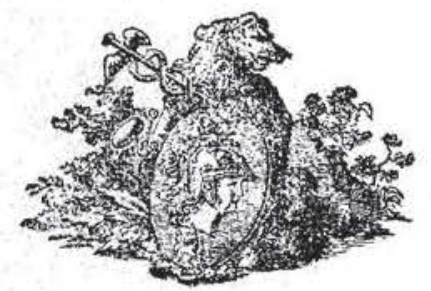

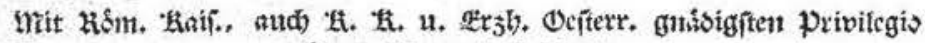
น́ber gefammite serblanos.

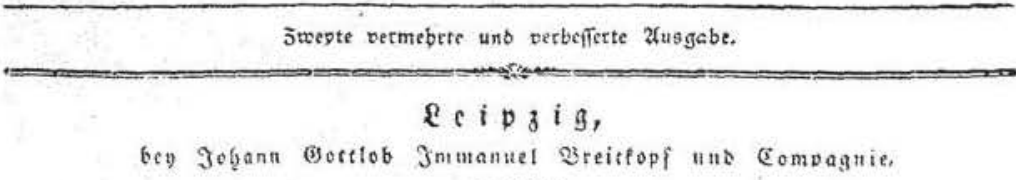

1793.

Abb. 2: Titelblatt: Johann Christoph Adelung, Grammatisch=kritisches Wörterbuch der Hochdeutschen Mundart, mit beständiger Vergleichung der übrigen Mundarten. besonders aber der Oberdeutschen. Erster Theil, von A-E. 


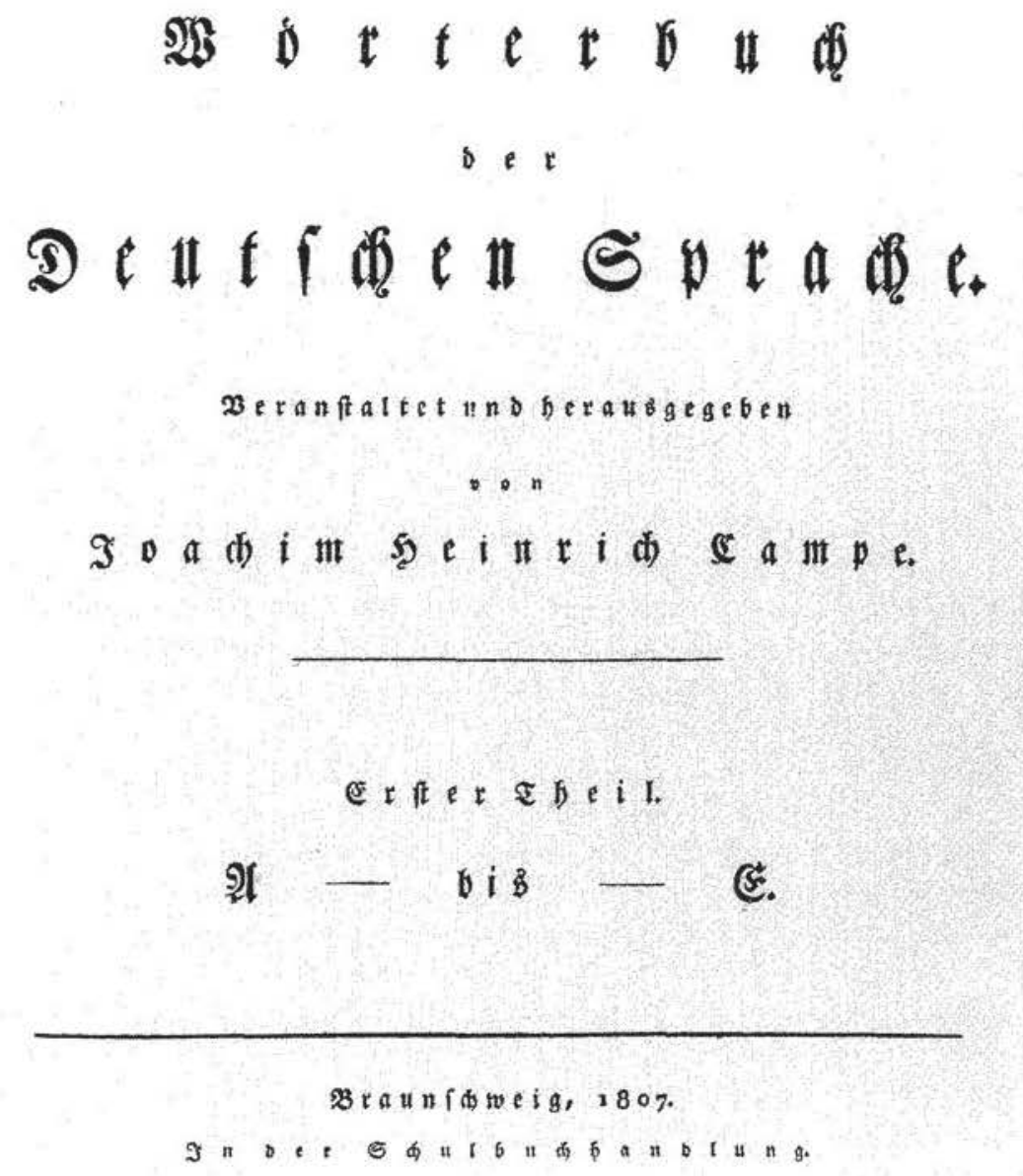

Abb. 3: Titelblatt: Johann Heinrich Campe, Wörterbuch der Deutschen Sprache. Erster Theil, von $\mathrm{A}-\mathrm{E}$. 


\title{
Spiegelungen der Rechtssprache in der Lexikografie
}

\author{
Haß-Zumkehr, Ulrike
}

Dieser Text wird über DuEPublico, dem Dokumenten- und Publikationsserver der Universität Duisburg-Essen, zur Verfügung gestellt.

Die hier veröffentlichte Version der E-Publikation kann von einer eventuell ebenfalls veröffentlichten Verlagsversion abweichen.

DOI: https://doi.org/10.1515/9783110932034.53

URN: urn:nbn:de:hbz:464-20190426-133120-6

Link: https://duepublico.uni-duisburg-essen.de:443/servlets/DocumentServlet?id=48651

Rechtliche Vermerke:

Ulrike Haß dankt dem De Gruyter Verlag für die freundliche Genehmigung, diesen Text in der Verlagsversion online veröffentlichen zu dürfen. Zudem dankt Frau Haß den Herausgebern für die Aufnahme des Beitrags in den Sammelband.

Quelle: In: Ulrich Kronauer / Jörn Garber (Hgg.): Recht und Sprache in der deutschen Aufklärung. Berlin/Boston: De Gruyter (Hallesche Beiträge zur Europäischen Aufklärung; 14), 2002, S. 53-71. - Online-ISBN 9783110932034 